\title{
DIGITAL TECHNOLOGY Using mobile ECG devices to increase detection of atrial fibrillation across a range of settings in south London
}

\author{
Authors: Alex Lang, ${ }^{\mathrm{A}}$ Faye Edwards, ${ }^{\mathrm{B}}$ Dominic Norton, ${ }^{\mathrm{C}}$ Laura Semple ${ }^{\mathrm{D}}$ and Helen Williams ${ }^{\mathrm{E}}$
}

The NHS Long Term Plan aims to transform how we tackle cardiovascular disease by improving the detection and treatment of high-risk conditions. One in five strokes are linked to atrial fibrillation (AF) and it is estimated that 500,000 people in the UK have undiagnosed AF. To increase detection of AF, in 2017 NHS England commissioned the Academic Health Science Networks to procure 6,000 mobile electrocardiography (ECG) devices, which were distributed to community settings across the county. The Health Innovation Network as the Academic Health Science Network for south London was responsible for the distribution of approximately 400 mobile ECG devices to a range of settings. A total of 14,835 pulse rhythm checks were performed, detecting 597 people with possible AF. This project provides insight into effectiveness of a wide range of settings in providing opportunistic testing for AF using mobile ECG devices.

KEYWORDS: Atrial fibrillation, stroke, mobile ECG devices

\section{Introduction}

The NHS Long Term Plan aims to transform how we tackle cardiovascular disease (CVD) in England. Improving the detection and treatment of high-risk conditions has the potential to unlock considerable health gains. Stroke is the fourth leading cause of death in the UK with approximately 100,000 people having a stroke each year. ${ }^{1}$ This human burden is mirrored by the cost to treat stroke, accounting for approximately $3-5 \%$ of all healthcare expenditure. ${ }^{2}$

Atrial fibrillation (AF) contributes to one in five strokes in the UK and is associated with greater disability and mortality that non AF-related strokes. ${ }^{1}$ While two-thirds of people with AF experience symptoms, one-third do not, with many only becoming aware of the condition when they have a stroke. ${ }^{3,4} \mathrm{AF}$ is relatively easy to diagnose, and treatment with anticoagulants can reduce risk of stroke by two-thirds. ${ }^{5}$ It is estimated that up to 500,000 people in

Authors: A project manager, Health Innovation Network, London, UK; ${ }^{\text {B }}$ project manager, Health Innovation Network, London, UK; ${ }^{C}$ assistant project manager, Health Innovation Network, London, UK; D programme director, Health Innovation Network, London, UK; Eclinical adviser for atrial fibrillation, AHSNs, and clinical director for atrial fibrillation, Health Innovation Network, London, UK the UK have undiagnosed AF. ${ }^{6}$ This leads to significantly increased risk of stroke, heart failure and renal disease and increases mortality when compared to those without $\mathrm{AF}^{7}$. With an ageing population, it is predicted that the number of people aged $>55$ years living with AF will more than double by $2060 .^{8}$

The UK National Screening Committee currently advises against screening for $\mathrm{AF}$ in the over $65 \mathrm{~s}$, concerned whether screening asymptomatic people has the same benefit of screening those with symptoms. ${ }^{9}$ Guidance from the European Society of Cardiology suggests systematic screening may be considered in those over the age of 75 years or at a high risk of stroke, with opportunistic screening recommended for those over 65 years. ${ }^{10}$ In general practice, opportunistic testing for AF was found to be more effective than routine care, and more cost-effective than systematic screening, based on the modelling for the cost of prevented strokes. ${ }^{11}$ Despite disagreement on whether to screen for $\mathrm{AF}$ in asymptomatic groups, some experts advocate testing for AF, and in some areas, this may be incentivised. This project helps inform the roll out of programmes where health services choose to run them. The authors recognise that the evidence around screening for AF will become clearer after the publication of 'The SAFER - Screening for AF Study', which is due to complete in 2026 and aims to determine whether systematic AF screening in over 65-year-olds in general practice is beneficial or not.

To reduce the incidence of $\mathrm{AF}$-related stroke through the increased detection of AF, in 2017 NHS England commissioned the 15 Academic Health Science Networks (AHSNs) to procure 6,000 mobile electrocardiography (ECG) devices, for distribution to community settings across the county. This paper outlines the experience and learning from one AHSN, aiming to review the feasibility of using mobile ECG devices in a range of settings for opportunistic testing for asymptomatic groups at increased risk of AF. It also aims to provide insight into the effectiveness of different healthcare and non-healthcare settings at detecting possible AF.

\section{Methods}

The Health Innovation Network (HIN) as the AHSN for south London was responsible for the distribution of 400 mobile ECG devices across the 12 boroughs of south London. Of the ECG devices available through the project, three-quarters of devices selected by HIN were Kardia Mobile (Alivecor) a credit card sized, single-lead ECG device that uses ultrasonic signal that is received via the microphone of a compatible smartphone or tablet. The app has a built-in AF detection algorithm that provides an instant 
interpretation and PDF of the ECG. The remaining devices were WatchBP Home A (Microlife Health Management), an automated blood pressure sphygmomanometer with a built-in AF algorithm. Systematic review and meta-analysis found both types of devices to have improved sensitivity and specificity compared to pulse palpation (Table 1)..$^{12}$

Expressions of interest were invited from south London clinical commissioning groups and local partners with a clear vision of how these devices would be used to test high numbers of people at increased risk of AF. Several additional settings were included during the project as interest in the devices spread, ensuring that mobile ECG devices were used in a range traditional and some more novel settings. Devices were gradually rolled out across the 12 boroughs of south London between January 2018 and March 2019.

HIN provided settings with hands-on, face-to-face training for the ECG devices and a range of supporting resources, which included the AHSN's project guidance covering a range of considerations for their use. The training and recommendations for use were adapted for the type of device received and for the setting in question. Settings were selected based on their potential for the high numbers of individuals at increased risk of AF they worked with, although a small number of additional novel settings were included that worked with groups at lower risk of AF, to assess the feasibility in such settings. Settings were recommended to offer pulse rhythm checks using the mobile ECG devices to opportunistically test all people deemed at increased risk of AF that they encountered. Specifically, this group at increased risk was identified as all those aged $>65$ years, or those aged $<65$ years with pre-existing cardiovascular disease risk factors, such as obesity and hypertension. Some settings were already offering pulse rhythm checks to individuals at lower risk of AF, for example during NHS health checks in individuals aged 40 years and over. In such cases where pulse checks were already being offered, manual pulse palpation was replaced by a pulse rhythm check using a mobile ECG device. Due to the increased sensitivity and specificity of both Kardia Mobile and WatchBP over manual pulse palpation, users were instructed to use devices as first line for pulse rhythm checks, rather than using them once an irregular pulse was detected manually.

Settings were instructed to test each person only once using the mobile ECG device. Exceptions to this were if they received an unreadable result from Kardia Mobile, which was usually due to noise interference and common in very loud environments. In these cases, users were advised to test in a quieter area where possible or revert to manual pulse checks where feasible. At the time of use, an unclassified result on Kardia Mobile was reported when the subject was tachycardic (heart rate $>100$ beats per minute (bpm))

Table 1. Meta-analysis pooled data assessing accuracy of electrocardiography device against pulse palpation

Sensitivity (95\% CI)

Pulse palpation
Blood pressure
machine

Smartphone application

$\mathrm{CI}=$ confidence interval. or bradycardic (heart rate $<50 \mathrm{bpm}$ ), regardless of heart rhythm. In such cases users were advised to wait a few minutes and then retest up to twice. If unclassified readings persisted, users were advised to treat in the same way as a possible AF finding. In such cases, a PDF of any abnormal traces detected using Kardia Mobile could be printed or emailed for advice from a general practitioner (GP), or appropriately trained healthcare professional, depending on the setting. Possible AF highlighted by WatchBP were treated the same way as if an irregular pulse had been detected manually. WatchBP was used for non-healthcare settings without an NHS Mail email address which was required to securely transfer the PDF of the trace from Kardia Mobile. As each device only identifies possible AF, pathways for onward referral for 12-lead ECG to confirm diagnosis were confirmed with each setting, ensuring timely and appropriate onward referral. Kardia Mobile usage was captured and reported to HIN monthly through the national AHSN project. WatchBP usage was collected manually by users and reported monthly to HIN by the lead from each setting.

\section{Results}

All 400 mobile ECG devices were distributed across the 12 boroughs of south London between January 2018 and March 2019, with the majority being deployed between May 2018 and March 2019. A total of 14,835 pulse rhythm checks were performed using both mobile ECG devices, detecting 597 people with possible AF (Table 2). Kardia Mobile was used to test 10,413 people, detecting 537 people with possible AF. WatchBP was used to perform 4,422 checks, finding 60 people with possible AF. As only $86 \%$ of Kardia Mobile users registered their devices with the AHSN, any activity performed by the remaining $14 \%$ of users $(42$ Kardia Mobile devices) that were not registered was not captured, which would have increased these numbers further. A breakdown of usage by device can be seen in Table 3. Device usage was fed back to users each month and learning was collected, allowing training materials and processes to be regularly improved. Device settings were grouped to enable interpretation into effectiveness of different settings at identifying possible AF.

\section{Discussion}

This study supports that using Kardia Mobile and WatchBP to opportunistically test groups at increased risk of AF is feasible across a range of different healthcare and non-healthcare settings, allowing more of the population to receive pulse rhythm checks to detect possible AF. Testing across these settings delivered 14,835 pulse rhythm checks to individuals who may otherwise have gone untested. Five-hundred and ninety-seven people were detected with possible AF who could be referred for 12-lead ECG and initiation of anticoagulation where appropriate, which reduces risk of stroke by two-thirds. ${ }^{5}$

A systematic review of screening for new AF reviewed thirty studies, including over 122,000 individuals. Undiagnosed AF was found in $1 \%$ of the overall population (one in 100) and $1.4 \%$ in those aged 65 years or older (one in 71). ${ }^{13}$ By using mobile ECG devices across a wide range of settings, it allows an estimation of the numbers of people needed to test in each setting to detect possible AF. Perhaps unsurprisingly, settings which included visiting housebound older people (one possible AF in seven people tested), and those seen in a range of specialist hospital-based medical outpatient clinics (one possible AF in 10 people tested), and GP 
Table 2. The number of pulse rhythm checks and possible atrial fibrillation findings by setting

\begin{tabular}{|c|c|c|c|c|c|}
\hline Setting & Device type & $\begin{array}{l}\text { Pulse rhythm } \\
\text { checks }\end{array}$ & Possible AF & $\begin{array}{l}\text { Detection } \\
\text { prevalence }\end{array}$ & $\begin{array}{l}\text { Detection } \\
\text { rate }\end{array}$ \\
\hline Older persons community teams & Kardia Mobile & 369 & 53 & $14.3 \%$ & 1 in 7 \\
\hline Hospital outpatient clinics & Kardia Mobile & 667 & 67 & $10.0 \%$ & 1 in 10 \\
\hline GP practices & Kardia Mobile & 4,494 & 264 & $5.9 \%$ & 1 in 17 \\
\hline Community podiatry & Kardia Mobile & 767 & 35 & $4.6 \%$ & 1 in 22 \\
\hline Fire Safe and Well & WatchBP & 25 & 1 & $4.0 \%$ & 1 in 25 \\
\hline Mental health & Kardia Mobile & 1,182 & 45 & $3.8 \%$ & 1 in 26 \\
\hline Community therapy teams & Both & 404 & 13 & $3.2 \%$ & 1 in 31 \\
\hline Community nursing teams & Both & 549 & 14 & $2.6 \%$ & 1 in 39 \\
\hline Pharmacy & Both & 2,820 & 57 & $2.0 \%$ & 1 in 49 \\
\hline Public health events & WatchBP & 2,393 & 44 & $1.8 \%$ & 1 in 54 \\
\hline Urgent Care & Kardia Mobile & 391 & 3 & $0.8 \%$ & 1 in 130 \\
\hline Prison & WatchBP & 774 & 1 & $0.1 \%$ & 1 in 774 \\
\hline Total & & 14,835 & 597 & $4.0 \%$ & 1 in 25 \\
\hline
\end{tabular}

practices (one possible AF in 17 people tested) found the greatest prevalence of possible AF.

Podiatrists are ideally placed to carry out opportunistic checks for undiagnosed AF as part of a routine podiatry assessment and when encountering visual symptoms that suggest an underlying circulatory disorder. The detection rate in community podiatry (one possible AF in 22 people tested) may be due to patients being older, and often with diabetes and existing cardiovascular risk factors. People with a serious mental illness have a life expectancy 15-20 years less than the general population, and are nearly twice as likely to suffer a stroke as the general population. ${ }^{14,15}$ This may, in part, explain the findings of one possible AF for every 26 people tested and supports further exploration of opportunistic testing in mental health settings.

Mobile ECG devices were used in several adult public health settings including AF awareness events, third sector health improvement services offering health checks to the general public, alongside activity centres for older people and a pop-up health shop offering health checks to adults of any age. The younger reported population within the section of the prison that the checks were performed in may explain the low detection rate observed in this setting. Although only one possible AF was detected in 774 people in the prison population, using WatchBP devices in prisons or sections of prisons with an older population at greater risk may increase the detection rate, and prison should not be discounted where this is the case. Using Kardia Mobile was not an option as the accompanying smart phone or tablet was not permitted within prisons. In an urgent care centre, a total of 391 people were tested over a 4 -week period by two student nurses, detecting three individuals with possible AF who were followed up in the hospital's rapid AF clinic. Testing for AF in urgent care with Kardia Mobile was feasible and acceptable to staff and service users. The younger population (mean age of 40 years) may account for the low detection rate observed in this setting. The lower proportion of possible AF detected by users of WatchBP, when compared to Kardia Mobile, may be explained by these devices being predominantly used by non-healthcare settings and often targeting adults of any age.

However, it is important to recognise limitations of this study. The small sample sizes in some settings limits the reliability of findings which need to be interpreted with caution. While users were discouraged from testing people who already had a diagnosis of $A F$, it's not possible to ensure this advice was always followed. The self-reporting of WatchBP usage also allows room for error. Data is not available on how many of those identified with possible AF went on to receive an AF diagnosis and treatment. Further investigation is therefore required to confirm the predicted conversion rate from possible AF to confirmed AF.

A limitation of Kardia Mobile is that it works through a free app which needs to be downloaded on smart phone or tablet. While some healthcare professionals had access to a work device which can be used alongside Kardia Mobile, many did not. This led to some people using their personal devices, and many unable to take part in the project. Some settings purchased a device

Table 3. A breakdown of usage by device

\begin{tabular}{llllll} 
& Number of devices & Pulse rhythm checks & Possible AF & Detection prevalence & Detection rate \\
Kardia Mobile & 300 & 10,413 & 537 & $5.1 \%$ & 1 in 19 \\
WatchBP & 100 & 4,422 & 60 & $1.4 \%$ & 1 in 73 \\
Total & 400 & 14,835 & 597 & $4.0 \%$ & 1 in 25 \\
\hline AF = atrial fibrillation. & & &
\end{tabular}


specifically for their Kardia Mobile, which increased usage. There was a noticeable variation in the digital literacy and confidence of users, making Kardia Mobile a challenge to embed in some areas. A limitation of WatchBP is that because the blood pressure is taken three times and then reports the average of these three, it takes longer to perform the measurement than using a traditional sphygmomanometer. For this reason, settings that had a very short appointment time with patients reported it difficult to embed these devices into their clinical practice.

Effective communication of positive findings to those being tested is key to reduce anxiety and written information on AF was provided to aid this process. This study and the work of the AHSN AF programme has increased the focus on AF, leading to pathway redesign in some boroughs and interventions to optimise the treatment of AF. The high prevalence of possible AF in housebound older people has prompted a further study focusing on the detection and treatment of $\mathrm{AF}$ in this population that is at risk of suboptimal treatment. ${ }^{16}$

For every 25 people diagnosed with AF and appropriately treated with anticoagulation, one stroke is prevented, saving an average of $£ 46,039$ per stroke in health and social care costs over 5 years. ${ }^{17}$ Any estimated healthcare savings need to be balanced against the cost of the opportunistic testing programme, including the cost of the mobile ECG devices, the cost of confirmatory 12-lead ECGs and the cost of the peoples' time to undertake the additional testing. While these costs were not estimated in this project, this is an important consideration when deciding whether to pursue opportunistic testing for AF in each setting.

\section{Conclusion}

These findings suggest that the greatest detection rates are to be found in testing groups of older people and those with existing CVD. It is feasible and effective to detect possible AF in several novel settings, such as community podiatry, mental health services and community therapy teams, which warrants further exploration. Manual pulse palpation in these settings may also be beneficial in areas where mobile ECG devices are not available. Although these innovative ECG devices are easy to use and popular with patients, they are only effective within the context of the whole patient pathway. A clear local process that enables timely investigation, diagnosis and treatment is paramount if a reduction in AF-related strokes is to be achieved. The NHS Long Term Plan advocates increased working with voluntary sector partners, community pharmacists and GP practices to provide wider opportunities for the public to test for the high-risk conditions. By estimating the possible AF detection rates, this paper highlights some additional settings which may be equally feasible and as effective to test for AF which warrants further exploration as a means of reducing the incidence of AF-related stroke through increased detection of AF.

\section{Acknowledgements}

The authors would like to acknowledge the AHSN and all the local partners who took part in the project.

\section{References}

1 Sentinel Stroke National Audit Programme. CCG/LHB results portfolio for April 2016-March 2017. London: King's College London. www.strokeaudit.org/results/Clinical-audit/Clinical-CCG-LHB-LCG. aspx [Accessed 23 May 2019].
2 Saka O, McGuire A, Wolfe C. Cost of stroke in the United Kingdom. Age Ageing 2009;38:27-32.

3 Healey JS, Connolly S], Gold MR et al. Subclinical atrial fibrillation and the risk of stroke. N Engl J Med 2012;366:120-9.

4 Borowsky LH, Regan S, Chang Y et al. First diagnosis of atrial fibrillation at the time of stroke. Cerebrovasc Dis 2017:43:192-9.

5 Björck S, Palaszewski B, Friberg L, Bergfeldt L. Atrial fibrillation, stroke risk, and warfarin therapy revisited: a population-based study. Stroke 2013;44:3103-8.

6 Public Health England: Atrial fibrillation prevalence estimates in England: Application of recent population estimates of $A F$ in Sweden. London: Public Health England, 2017. www.gov.uk/ government/publications/atrial-fibrillation-prevalence-estimatesfor-local-populations [Accessed 23 May 2019].

7. Odutayo A, Wong CX, Hsiao AJ et al. Atrial fibrillation and risks of cardiovascular disease, renal disease, and death: systematic review and meta-anaysis. BMJ 2016;354:14482.

8. Krijthe BP, Kunst A, Benjamin EJ et al. Projections on the number of individuals with atrial fibrillation in the European Union, from 2000 to 2060. Eur Heart J 2013;34:2746-51.

9 UK National Screening Committee. Screening for atrial fibrillation in people aged 65 years and over. Edinburgh: Royal College of Physicians of Edinburgh, 2014.

10 Camm AJ, Lip GYH, De Caterina R et al. 2012 focused update of the ESC guidelines for the management of atrial fibrillation: an update of the 2010 ESC guidelines for the management of atrial fibrillation. Developed with the special contribution of the European Heart Rhythm Association. Eur Heart J 2012;33:271947.

11 Hobbs FDR, Fitzmaurice DA, Mant ] et al. A randomised controlled trial and cost effectiveness study of systematic screening (targeted and total population screening) versus routine practice for detection of atrial fibrillation in people over 65 years and over. The SAFE study. Health Technol Assess 2005;9:iii-iv, ix-x, 1-74.

12 Taggar JS, Coleman T, Lewis S et al. Accuracy of methods for detecting an irregular pulse and suspected atrial fibrillation: a systematic review and meta-analysis. Eur J Prev Cardiol 2016;23:1330-8.

13 Lowres N, Neubeck L, Redfern J, Freedman SB. Screening to identify unknown atrial fibrillation: a systematic review. Thromb Haemost 2013;110:213-22.

14 Chesney E, Goodwin GM, Faisal F. Risks of all cause and suicide mortality in mental disorders: a meta-review. World Psychiatry 2014;13:153-60.

15 Public Health England: Severe mental illness (SMI) and physical health inequalities: briefing. London: Public Health England, 2018. www.gov.uk/government/publications/severe-mental-illness-smiphysical-health-inequalities/severe-mental-illness-and-physicalhealth-inequalities-briefing [Accessed 23 August 2019].

16 Van Walraven C, Hart RG, Connolly S et al. Effect of age on stroke prevention therapy in patients with atrial fibrillation: the atrial fibrillation investigators. Stroke 2009:40:1410.

$17 \mathrm{Xu} \mathrm{XM}$, Vestesson E, Paley L et al. The economic burden of stroke care in England, Wales and Northern Ireland: Using a national stroke register to estimate and report patient-level health economic outcomes in stroke. Eur Stroke J 2018;3:82-91.

Address for correspondence: Mr Alex Lang, Health Innovation Network, Ground Floor, Minerva House, 5 Montague Close, London SE1 9BB, UK.

Email: alexlang@nhs.net 